\title{
Structural analysis, ferroelectric and dielectric properties of single component organic multifunctional materials
}

\author{
Rekha Kumari, T. N. Guru Row \\ Solid State and Structural Chemistry Unit, Indian Institute of Science, Bengaluru, India \\ rekhakumari@iisc.ac.in; gururow@iisc.ac.in
}

Ferroelectric effects are observed in wide variety of materials such as composite ceramics, solids, polymers, crystals and liquid. Various inorganic compounds, such as, barium titanate (BaTiO3), potassium dihydrogen phosphate (KDP), Lithium niobate (LiNbO3), lead zirconate titanate (PZT) etc are well known for decades. On the other hand, organic ferroelectrics in recent times have attracted considerable attention in the material science due to their potential applications as ferroelectric random-access memories (FeRAM), non-volatile random-access memories (NVRAMs) and dynamic random-access memories (DRAMs) etc. However, single component organic compounds that show multifunctional properties have hardly investigated and very few reports are available in the literature.

In the present investigation, we report the crystal structures of two N-Benzylideneaniline analogues (BOA and HBOA) and analyse their hydrogen bonding interactions, second harmonic generation efficiency, ferroelectric and dielectric properties. Both the molecules are crystallized in a non-centrosymmetric with monoclinic space group $P 2_{1}$ and stabilized by strong intermolecular interactions through $\mathrm{O}-\mathrm{H} . . . \mathrm{O}$ and $\mathrm{N}-\mathrm{H} . . . \mathrm{O}$ hydrogen bonds. SHG activity of BOA and HBOA also confirms their non-centrosymmetric nature which is prerequisite characteristics for ferroelectric materials. SHG efficiency was found to be $68 \mathrm{mv}$ and $140 \mathrm{mv}$ for BOA and HBOA respectively with respect to KDP (75mv, standard). Further large thermal hysteresis was observed from the DSC scan of BOA and HBOA at the range of $100{ }^{\circ} \mathrm{C}$ and $87{ }^{\circ} \mathrm{C}$ respectively. Furthermore, both the compounds have shown significant reversible mechanofluorochromic (MFC) behaviour upon grinding and fuming. The reversible MFC behaviour was confirmed by cognate techniques like powder X-ray diffraction (PXRD), thermal analysis and fluorescence studies. Both the systems were further investigated to demonstrate the structure-property relationship for realizing their ferroelectric behaviour by PUND (positive up and negative down). Interestingly, both the molecules are fascinating ferroelectric behaviour at high electric field and tolerate upto 181$182 \mathrm{kV} / \mathrm{cm}$ without any breakdown (upto instrument limitation). The origin of ferroelectricity has been observed due to the proton transfer in both the systems (Shown in Fig. 1). The dielectric loss-factor was measured in a wide range of frequency at room temperature. High dielectric constant and low loss of energy indicate that the materials can be good candidate for energy storage application. The combination of findings provides a potential perspective for designing new organic multifunctional materials for the applications in light displaying and memory devices.

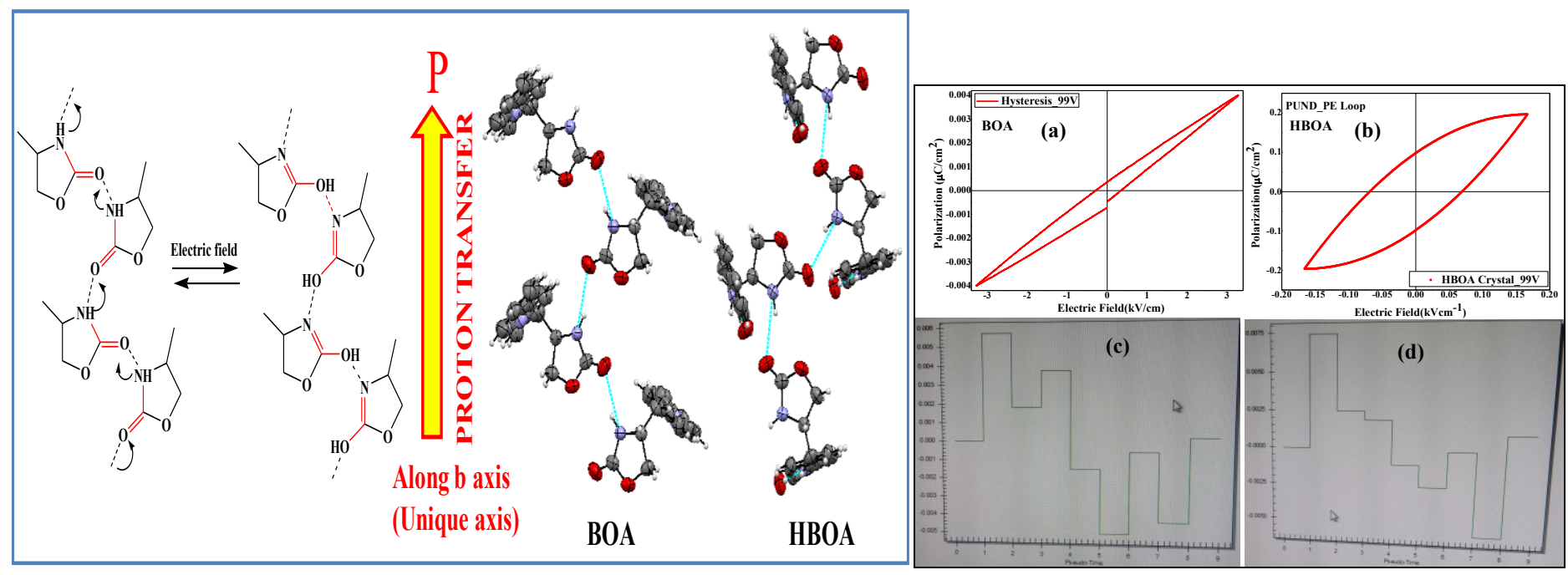

Figure 1. The crystal polarity can be inverted by proton transfer over a hydrogen bond and simultaneous interconversion of the single and double bonds, which are specified in red. The dotted lines indicate the proton transfer paths over the intermolecular N-H...O hydrogen bonds. The thick arrow indicates the electric polarity of each hydrogen-bonded chain. PE loop and PUND results are at right side. 


\section{Poster Session}

[1] R. Kaur, B. Ponraj, D. Swain, K. B. R. Varma, and T. N. Guru Row, "Anomalous Dielectric Features and Structural Implications in Gallic Acid Isoniazid Cocrystal Mediated by Lattice Water”, Cryst. Growth Des., 2015, 15, 4171-4176.

[2] R. Kumari, R. Seera, A. De, R. Ranjan, T. N. G. Row, “Organic Multifunctional Materials: Second Harmonic, Ferroelectric, and Dielectric Properties in N-Benzylideneaniline Analogues", Cryst. growth Des., 2019, 5934-5944.

Keywords: Thermal hysteresis, Mechanofluorochromic, P-E hysteresis loops, Dielectric properties. 